Alma Mater Studiorum - Università di Bologna DEPARTMENT OF ECONOMICS

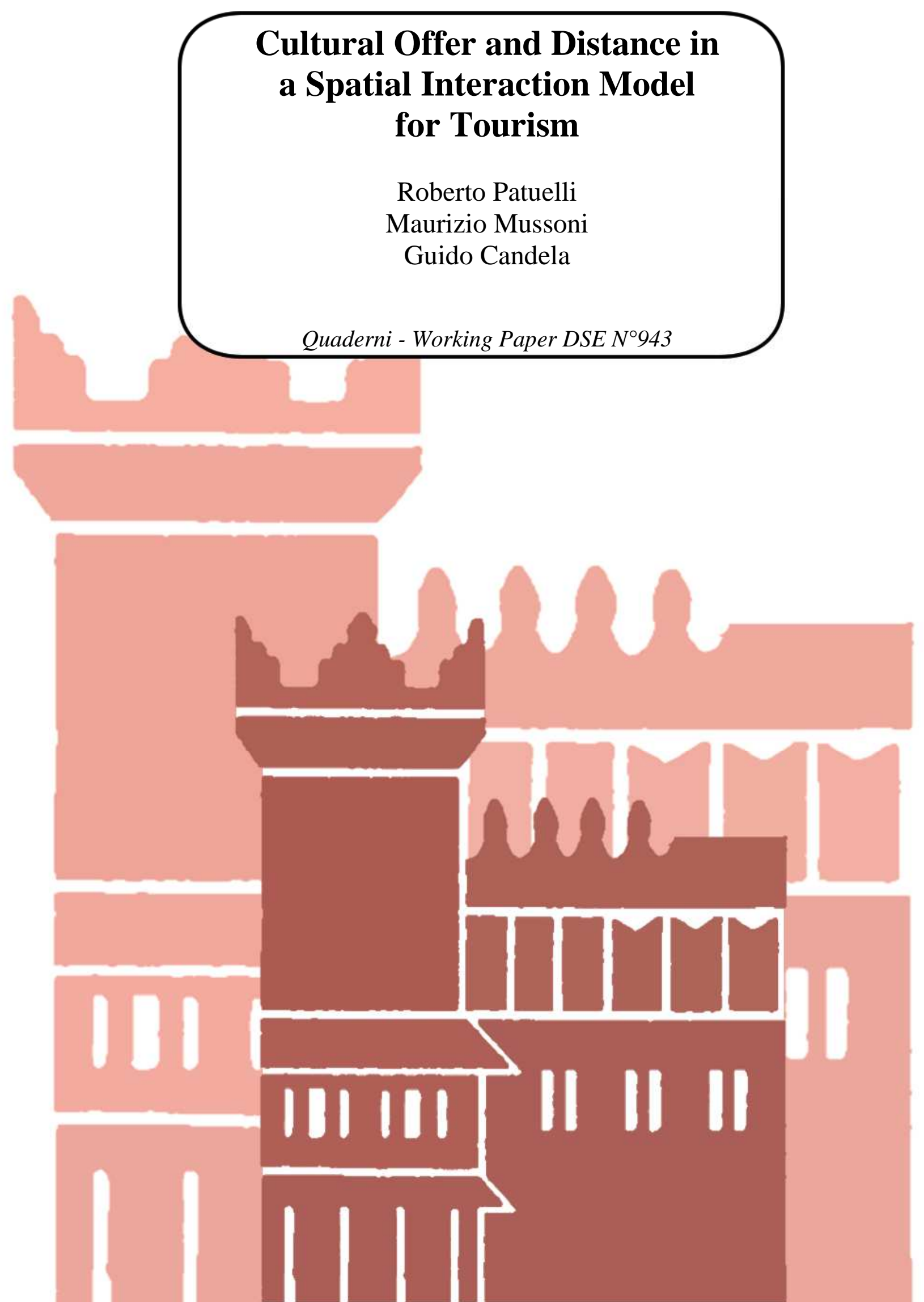




\title{
Cultural Offer and Distance in a Spatial Interaction Model for Tourism
}

\author{
Roberto Patuelli ${ }^{1}$, Maurizio Mussoni ${ }^{2}$ and Guido Candela $^{3}$ \\ ${ }^{1}$ Department of Economics, University of Bologna, Italy; The Rimini Centre for Economic \\ Analysis (RCEA), Italy. Email: roberto.patuelli@unibo.it \\ ${ }^{2}$ Department of Economics, University of Bologna, Italy; The Rimini Centre for Economic \\ Analysis (RCEA), Italy \\ ${ }^{3}$ Department of Economics, University of Bologna, Italy.
}

This version: 07/05/2014

\begin{abstract}
Culture is more and more considered as an important driver of tourism. However, it is critical, for policymakers, to evaluate the potential returns from investments in culture and generally cultural offer, in particular in multiregion settings with a potentially inefficient distribution of cultural offer. Our paper focuses on the role of distance (between the tourist's origin and destination regions) in mediating the tourism impact of cultural offer. This research question is investigated by means of a spatial interaction model, applied to the case of Italian domestic tourism. We find that distance indeed matters: a destination's endowment in culture appears to be more attractive for long-distance tourists, while an origin region's endowment seems to dinsincentivate long-distance trips to a greater extent.
\end{abstract}

Keywords: cultural offer; domestic tourism; spatial interaction model; distance; spatial competition.

JEL codes: C23; L83; R12; Z10. 


\section{Introduction}

Culture is nowadays more and more considered as an important driver of tourism and a suitable tool for alleviating the effects of seasonality (see, e.g., Cuccia and Rizzo, 2011). However, it is critical, for policymakers, to evaluate the potential returns from investments in culture and generally cultural offer. This is particularly true in a multiregion setting where each region may devise plans for its cultural offer, in a potentially inefficient scenario of competing local endowments in culture (Candela et al., 2014). In this regard, Patuelli et al. (2013) have recently shown, by means of a spatial interaction model estimated for Italian domestic tourism, that a region's positive effect on incoming tourism flows deriving from the acquisition of a UNESCO certification may be offset by further UNESCO certifications acquired by nearby regions, because of spatial competition. The strength of such spatial competition has been shown, by Patuelli et al. (2013), to depend on the definition of 'nearby' regions, for example in terms of their distance from the destination.

One may then wonder to what extent such results apply to tourists that face different opportunity costs for their travel, typically measured, in spatial interaction models, by the distance between the tourist's origin and the destination. In other words: is the cultural offer of regions relevant for all tourists, near and far away?

Our paper focuses on this question. In particular, our research question may be subdivided into two subquestions, pertaining to the origin and the destination of tourists:

Q1: If the origin region's cultural offer influences the propensity of inhabitants to travel (e.g., negatively, because of substitution between recordable tourism and excursionism), is such effect homogeneous over distance?

Q2: If the destination region's cultural offer positively influences incoming tourism flows, is such positive effect homogeneous over distance?

Relying on Patuelli et al.'s (2013) empirical framework and data set, the above research questions are tested by means of a spatial interaction model, applied to the case of Italian domestic tourism (which accounts for up to 88 per cent of arrivals, at the regional scale; Massidda and Etzo, 2011). Empirically, interaction terms between the origin and destination region's evaluations of cultural offer and the distance variable are used to evaluate the potential heterogeneity of the effect of cultural offer.

Moreover, one could be interested in investigating how spatial competition (or its opposite, spatial complementarity) effects induced by the cultural offer of nearby regions affects tourism flows along the lines sketched above. For instance: Is spatial competition between destinations homogeneously strong or does it differ for short- and long-distance tourists? Therefore, similarly to questions $Q 1$ and $Q 2$, we test by means of spatial lag variables the sensitivity of our model to the evaluation of cultural offer in neighbouring regions and distance.

We find that distance indeed matters for the effect of cultural offer on tourism: a destination's endowment in culture appears to be more attractive for long-distance tourists, while an origin region's endowment seems to dinsincentivate long-distance trips to a greater extent. Similar results (i.e., effects increasing with distance) are found for the cultural endowment of neighbouring regions.

\section{Methods}

The spatial interaction model (see Haynes and Fotheringham, 1984; Sen and Smith, 1995), is a modelling framework typically used in many fields of study to explain dyadic flows (i.e. between an origin and a destination). In the case of tourism, it has often been used to 
investigate the flows of tourists between regions or countries (e.g., Uysal and Crompton, 1985; Witt and Witt, 1995; Khadaroo and Seetanah, 2008).

Similarly to previous applications of the spatial interaction model to tourism, we model bilateral tourism flows (arrivals in region $j$ from region $i ; T_{i j}$ ) as depending on a number of characteristics of the regions of origin (influencing outflows) and destination (affecting inflows) and the distance between them, representing a proxy of transportation/opportunity costs.

We rely on the empirical approach of Patuelli et al. (2013) for the choice of variables commonly used as push and pull factors (see, e.g., Sheldon and Var, 1985; Lim, 1997), such as regional GDP, population, price indices, crime indices, touristic specialization and deseasonalization.

With regard to the explanatory variables pertaining to the regions' cultural offer, we include: (i) public spending in recreational/cultural activities, which we take as an indicator of the local administrations' investment in attracting tourists; (ii) the average number of visitors per state museum, as a proxy of the quality of public museums; (iii) the number of tickets sold per inhabitant for theatrical and musical events, as a further indicator of the quality of public and private cultural events; and (iv) the number of UNESCO-certified World Heritage Sites (WHS), to identify the presence of points of attraction for the tourist.

The relevance of cultural offer/endowment for tourism has been investigated in many studies. Some studies suggest that cultural heritage and attractions are major tourism drivers (e.g., Herbert, 2001; Vietze, 2008), also because of their uniqueness and difficult transferability (Dritsakis, 2004). Other studies stress do not find cultural sites and attractions to effectively attract tourists (see, e.g., Cuccia and Cellini, 2007). Similar contradictory results are found, for example, for WHS designations.

We estimate a spatial interaction model for a 12-year panel (from 1998 to 2009) of tourism flows (arrivals) between all 20 Italian regions, including both origin-destination-pair and time fixed effects (FE). Following Santos Silva and Tenreyro (2006), the model is estimated in a Poisson-type framework, but employing a negative binomial specification to account for overdispersion. We can then write a baseline model [Model (1)] to be estimated as follows:

$$
T_{i j t}=\exp \left(\alpha_{i j}+y e a r_{t}+X_{i t}+C O_{i t}+L . C O_{i t}+X_{j t}+C O_{j t}+L \cdot C O_{j t}+D i s t_{i j}\right)+\varepsilon_{i j t},
$$

where $\alpha_{i j}$ identifies individual FE for the origin $i$ and destination $j$ pair, year ${ }_{t}$ is time $\mathrm{FE}, X$ and $C O$ are the sets of control and cultural offer variables, respectively, evaluated at both origin $i$ and destination $j$, and Dist is the geographical (centroid) distance between each region pair. The latter term, being time-invariant, is dropped during estimation because of the individual FE. To account for the influence of the spatial distribution of cultural offer on tourism flows, as in a competing destinations (Fotheringham, 1983) or trip-chaining framework, we compute, for the statistically significant cultural offer variables, their spatial lag counterparts $L . C O=\mathbf{W C O}$ by means of a row-standardized spatial weights matrix $\mathbf{W}$ based on rook contiguity (share border) (for details, see Patuelli et al., 2013).

In order to test the research questions outlined in Section 1, we augment Eq. (1) by considering interaction terms between the distance (Dist) and the cultural offer $(C O)$ variables (for both origins and destinations). Consequently, the resulting model [Model (2)] is the following:

$$
\begin{aligned}
& T_{i j t}=\exp \left[\alpha_{i j}+y e a r_{t}+X_{i t}+C O_{i t}+L . C O_{i t}+X_{j t}+C O_{j t}+L . C O_{j t}+D i s t_{i j}\right. \\
& \left.+\left(C O_{i t}+L . C O_{i t}+C O_{j t}+L . C O_{j t}\right) \times D i s t_{i j}\right]+\varepsilon_{i j t} .
\end{aligned}
$$


For the purpose of model simplicity, interaction terms are applied only to statistically significant $C O$ variables. With regard to the functional specification of the distance deterrence factor, we test both power and exponential specifications, with the former resulting preferable, as well as polynomial specifications, with a quadratic polynomial being selected for Model 2 on the basis of the Akaike information criterion (AIC).

\section{Data}

We employ data entirely obtained from the Italian National Institute of Statistics (ISTAT), with the only exception of the distance variable, which is computed by means of GIS software, and the variable for the number of WHS, which is obtained directly from UNESCO's World Heritage Convention website (http://whc.unesco.org). Our dependent variable - arrivals per region subdivided by origin region - was obtained for the period 19982009 and is published in the 'Statistiche del Turismo' publication. The data, as in most countries, are collected from accommodation structures who have the obligation to communicate all arrivals to local public officials. The survey includes both traditional hotel accommodation and alternatives such as complementary accommodations and privately rented houses. All further variables are published in the following databases: 'Conti Economici Regionali', 'Prezzi al Consumo', and 'Banca Dati Territoriale per le Politiche di Sviluppo'. Table 1 provides basic information on the set of control variables used in the model as well as the set of cultural offer variables. All variables are taken in logs, aside from WHS and NonBath, which include a share of zeros.

\section{Results}

In Table 2, we report estimation results for both Model (1) and Model (2) defined in Section 2. We compute spatial lags for the cultural offer variables that are found to be statistically significant (at least for the origin or the destination) in a preliminary model estimation (not shown, available on request), that is, CultDem and WHS.

Model (1) presents standard (expected) results for the set of control variables. The level of prices of the restoration/accommodation sector (PricesH\&R) negatively influence inflows, while regions which deseasonalize (OffSeas) experience greater inflows. Violent crime levels (CrimVio) and polluted coasts (NonBath) appears to deter incoming tourism. Specialization in tourism (SpecTour) not only is obviously related to inflows, but also appears to increase the propensity to travel of residents, either according to an 'addiction to tourism' effect or as crowding out of tourists on residents.

With regard to the cultural offer variables, diffusion of shows is not significant, while public expenditure in events is significant for both origins and destinations, suggesting for the latter an economically relevant effect on incoming tourism flows for increases in the share of spending for public events. The variables for the quality of public museums and for the number of WHS have statistically significant and qualitatively similar effects on tourism: both of them influence inflows positively, but generate equally intense spatial competition, on the basis of the (competing) neighbours' efforts/endowment. We stress that this is an important result, already found for WHS in Patuelli et al. (2013), which seems to rule out, at least on average, the possibility of spatial complementarity effects, and suggests the need for coordination of cultural offer policies to avoid global inefficiencies induced by spatial competition (if we consider the state as the leading provider of public funds to regions) (Candela et al., 2014). Additionally, for WHS, a negative effect on outflows is also observed, 
suggesting possible substitution between tourism in accommodation structures and excursionism (daily trips without spending the night away from home) to nearby WHS attractions.

Table 1. Explanatory variables

\begin{tabular}{|c|c|c|}
\hline Variable & Description & Source \\
\hline & Control variables & \\
\hline GDP & Regional GDP (1-year lag, in logs) & ISTAT \\
\hline SpecTour & $\begin{array}{l}\text { Specialization in tourism (= share of value added generated from } \\
\text { accommodation and restaurants, commerce, transport, etc.) ( } 2- \\
\text { year lag, in logs) }\end{array}$ & ISTAT \\
\hline PricesH\&R & Price index for hotels and restaurants (in logs) & ISTAT \\
\hline Pop & Regional population (in logs) & ISTAT \\
\hline CrimDiff & $\begin{array}{l}\text { Small crime index (= thefts and robberies x 1,000 inhabitants) } \\
\text { (1-year lag, in logs) }\end{array}$ & ISTAT \\
\hline CrimVio & $\begin{array}{l}\text { Violent crime index (= violent crimes x 10,000 inhabitants) (2- } \\
\text { year lag, in logs) }\end{array}$ & ISTAT \\
\hline NonBath & $\begin{array}{l}\text { Coast unsuitable for bathing (= share of coast kms which are } \\
\text { unsuitable for bathing due to pollution) (1-year lag, in logs) }\end{array}$ & ISTAT \\
\hline OffSeas & $\begin{array}{l}\text { Deseasoning index (= overnight stays in off-season months } \mathrm{x} \\
\text { inhabitant })(1 \text {-year lag, in logs })\end{array}$ & ISTAT \\
\hline Dist & Distance between regional centroids (in km, in logs) & $\begin{array}{l}\text { Own } \\
\text { calculation }\end{array}$ \\
\hline ExpRecr & $\begin{array}{l}\text { Cultural offer variables } \\
\text { Share of public spending in recreational, cultural and religious } \\
\text { activities ( } 2 \text {-year lag, in logs) }\end{array}$ & ISTAT \\
\hline CultDem & $\begin{array}{l}\text { Cultural demand index (= visitors to state antiquities and arts } \\
\text { museums x institute) (1-year lag, in logs). }{ }^{1}\end{array}$ & ISTAT \\
\hline DiffShows & $\begin{array}{l}\text { Diffusion of theatrical and musical shows (= theatrical and } \\
\text { musical shows tickets sold x } 100 \text { inhabitants) (1-year lag, in } \\
\text { logs) }\end{array}$ & ISTAT \\
\hline WHS & Number of WHS (in logs) & UNESCO \\
\hline
\end{tabular}

Source: Modified from Patuelli et al. (2013).

Our findings above motivate the main analysis proposed in the paper, that is, a sensitivity analysis of what is found in Model (1) for the cultural offer variables with respect to distance. The latter is a critical variable in spatial interaction models (though it drops out here, because of the panel framework), and an even more particular one in tourism economics, given the unique possibility of travelling being perceived as leisure, and visiting distant destinations as exotic (therefore increasing individual utility instead of decreasing it). By interacting the distance variable with the cultural offer variables for museum quality and WHS, we estimate Model (2), whose results are again given in Table 2. A quadratic distance polynomial is used, which was chosen on the basis of $\chi^{2}$-based likelihood ratio tests.

1 CultDem values for Aosta Valley are set to zero for all years, due to the lack of state museums (source: Ministry of Cultural Heritage and Activities). For Trentino-Alto Adige/Südtirol and Sicily, missing values for more recent years (four and two, respectively) denote the passage of all state museums to other administrations, therefore they are set to the average of the previous years. 
Table 2. Empirical estimates

\begin{tabular}{|c|c|c|c|c|}
\hline & $\begin{array}{l}\text { Estimate (Std error) } \\
\text { Model (1) }\end{array}$ & p-value & $\begin{array}{l}\text { Estimate (Std error) } \\
\text { Model (2) }\end{array}$ & p-value \\
\hline & \multicolumn{4}{|l|}{ Control variables } \\
\hline GDP orig & $0.2013(0.3242)$ & 0.5346 & $0.1810(0.3219)$ & 0.5739 \\
\hline GDP dest & $-1.8308(0.3049)$ & $<0.0001$ & $-1.7217(0.2934)$ & $<0.0001$ \\
\hline SpecTour orig & $0.4589(0.0928)$ & $<0.0001$ & $0.3968(0.0898)$ & $<0.0001$ \\
\hline SpecTour dest & $0.3838(0.1041)$ & 0.0002 & $0.3027(0.1041)$ & 0.0036 \\
\hline PricesH\&R orig & $0.3512(0.2315)$ & 0.1294 & $0.3317(0.2218)$ & 0.1347 \\
\hline PricesH\&R dest & $-1.3196(0.2101)$ & $<0.0001$ & $-1.2957(0.2073)$ & $<0.0001$ \\
\hline Pop orig & $-0.0274(0.3764)$ & 0.9419 & $-0.4575(0.4163)$ & 0.2718 \\
\hline Pop dest & $1.3238(0.2396)$ & $<0.0001$ & $0.7733(0.2496)$ & 0.0019 \\
\hline CrimDiff orig & $0.0859(0.0471)$ & 0.0682 & $0.0619(0.0421)$ & 0.1415 \\
\hline CrimDiff dest & $0.0258(0.0252)$ & 0.3068 & $0.0021(0.0253)$ & 0.9339 \\
\hline CrimVio orig & $0.0569(0.0237)$ & 0.0165 & $0.0399(0.0237)$ & 0.0919 \\
\hline CrimVio dest & $-0.0495(0.0220)$ & 0.0244 & $-0.0714(0.0217)$ & 0.0010 \\
\hline NonBath orig & $-0.0109(0.0137)$ & 0.4268 & $-0.0126(0.0135)$ & 0.3500 \\
\hline NonBath dest & $-0.0304(0.0122)$ & 0.0127 & $-0.0342(0.0117)$ & 0.0035 \\
\hline OffSeas orig & $0.0527(0.0372)$ & 0.1563 & $0.0580(0.0357)$ & 0.1042 \\
\hline \multirow[t]{2}{*}{ OffSeas dest } & $0.3861(0.0415)$ & $<0.0001$ & $0.4013(0.0390)$ & $<0.0001$ \\
\hline & \multicolumn{4}{|l|}{ Cultural offer variables } \\
\hline ExpRecr orig & $0.1094(0.0549)$ & 0.0464 & $0.0926(0.0529)$ & 0.0803 \\
\hline ExpRecr dest & $0.1514(0.0411)$ & 0.0002 & $0.1537(0.0396)$ & 0.0001 \\
\hline CultDem orig & $-0.0330(0.0203)$ & 0.1044 & $-0.0447(0.0205)$ & 0.0288 \\
\hline CultDem orig $\times$ Dist & - & - & $1.2129(2.4575)$ & 0.6216 \\
\hline CultDem orig $\times$ Dist $^{2}$ & - & - & $-3.6915(2.0096)$ & 0.0662 \\
\hline L.CultDem orig & $0.0034(0.0324)$ & 0.9166 & $0.0051(0.0323)$ & 0.8750 \\
\hline L.CultDem orig $\times$ Dist & - & - & $-4.7181(2.4676)$ & 0.0559 \\
\hline L.CultDem orig $\times$ Dist $^{2}$ & - & - & $-0.7016(2.2965)$ & 0.7600 \\
\hline CultDem dest & $0.1971(0.0227)$ & $<0.0001$ & $0.1959(0.0258)$ & $<0.0001$ \\
\hline CultDem dest $\times$ Dist & - & - & $1.5574(1.9010)$ & 0.4126 \\
\hline CultDem dest $\times$ Dist $^{2}$ & - & - & $4.7725(1.9177)$ & 0.0128 \\
\hline L.CultDem dest & $-0.1846(0.0356)$ & $<0.0001$ & $-0.1703(0.0394)$ & $<0.0001$ \\
\hline L.CultDem dest $\times$ Dist & - & - & $-6.7227(3.6277)$ & 0.0639 \\
\hline L.CultDem dest $\times$ Dist $^{2}$ & - & - & $-4.6526(2.1619)$ & 0.0314 \\
\hline DiffShows orig & $0.0384(0.0338)$ & 0.2559 & $0.0358(0.0348)$ & 0.3040 \\
\hline DiffShows dest & $-0.0002(0.0275)$ & 0.9933 & $-0.0004(0.0256)$ & 0.9870 \\
\hline WHS orig & $-0.0568(0.0265)$ & 0.0321 & $-0.0615(0.0259)$ & 0.0175 \\
\hline WHS orig $\times$ Dist & - & - & $0.5615(1.8112)$ & 0.7565 \\
\hline WHS orig $\times$ Dist $^{2}$ & - & - & $0.3877(1.8159)$ & 0.8310 \\
\hline L.WHS orig & $-0.0742(0.0543)$ & 0.1719 & $-0.0893(0.0614)$ & 0.1456 \\
\hline L.WHS orig $\times$ Dist & - & - & $-7.4772(5.3453)$ & 0.1619 \\
\hline L.WHS orig $\times$ Dist $^{2}$ & - & - & $-5.8272(3.8406)$ & 0.1292 \\
\hline WHS dest & $0.2102(0.0297)$ & $<0.0001$ & $0.2137(0.0305)$ & $<0.0001$ \\
\hline WHS dest $\times$ Dist & - & - & $0.7072(2.1244)$ & 0.7392 \\
\hline WHS dest $\times$ Dist $^{2}$ & - & - & $2.3837(1.8600)$ & 0.2000 \\
\hline L.WHS dest & $-0.2718(0.0533)$ & $<0.0001$ & $-0.2807(0.0572)$ & $<0.0001$ \\
\hline L.WHS dest $\times$ Dist & - & - & $-10.8868(4.8843)$ & 0.0258 \\
\hline L.WHS dest $\times$ Dist $^{2}$ & - & - & $-0.6115(3.4816)$ & 0.8606 \\
\hline AIC & 90975 & - & 90866 & - \\
\hline $\mathrm{BIC}$ & 93778 & - & 93771 & - \\
\hline Res. dof & 3942 & - & 3926 & - \\
\hline McFadden's pseudo- $R^{2}$ & 0.2459 & - & 0.2475 & \\
\hline $\begin{array}{l}\text { ANOVA ( } \chi^{2} \text { LR test): } \\
\text { Model (1) vs Model (2) }\end{array}$ & - & - & 141.1737 & $<0.0001$ \\
\hline
\end{tabular}

Note: The distance variable (single term) drops out because of individual FE. Robust standard errors are applied. All explanatory variables are in logs. 
In Model (2), our results for the control variables remain virtually unchanged. We may then focus on the cultural offer variables. We find that the effect of public expenditure in events remains statistically significant and virtually unchanged numerically. At the same time, the diffusion of theatrical and musical shows remains non-significant. For museum quality (CultDem) and WHS, for which we include both spatial lag terms and - now interaction terms, the estimated effects are of greater complexity.

Generally, we find that the signs and significance levels found in Model (1) for the single terms are confirmed. Moreover, in three of eight cases (five is considering 10\% significance), distance appears to significantly interact with the variables studied. When statistically significant, the distance interaction terms show the same sign of the single term (i.e., CultDem or WHS). For example, in the case of destination regions, on the one hand distance appears to strengthen the positive (pull) effect of cultural offer; on the other hand, the same happens to spatial competition (the negative effect found for destinations' spatial lags).

Such complex interactive effects can be best inspected visually, for example by plotting the estimated marginal effects of the interacted cultural offer variables for different representative values of the distance variables. We use quintiles of Dist, and therefore plot five marginal effects for each of the variables concerned. In each graph, the top-right plot shows the effect of the independent variable on the dependent for the highest quantile of the distance variable (origin-destination pairs with greater geographical distances), while the bottom-left plot is for the lowest quantile. All graphs are on the scale of the response variable, providing, on the y-axis, the expected tourism flows values.

Because our findings for CultDem and WHS are qualitatively comparable, we limit ourselves to plotting the marginal effects for CultDem, while we provide the plots for WHS in the Appendix. In Figure 1, we plot the marginal effects for the interaction of the distance variable with the CultDem origin variables (CultDem orig and L.CultDem orig), while in Figure 2 we do the same for the destination variables (CultDem dest and L.CultDem dest).

With regard to the museums of the origin regions (CultDem orig), we see in Figure 1 that their quality's overall negative effect on outflows seen in Table 2 does not seem to vary dramatically over distance (as suggested by the only marginally significant interaction terms). On the other hand, despite thicker confidence intervals for the higher distance quintiles, the estimated effects appear to gradually flatten out or even reverse pendency (for L.CultDem orig). The numerical and graphical evidence suggests that the availability of higher quality state museums in relative proximity of one's residence region tends to disincentivate faraway trips. Quite logically, such effect can be expected to even become positive for the shortest distances, as these are the ones that correspond to the regions on which L.WHS orig is computed.

When inspecting the case of destination regions (Figure 2), similar and clearer attenuation effects can be observed. The positive effect of CultDem dest on incoming flows found in Models (1) and (2) is implied to be heterogeneous by the significant interaction terms. The marginal effect plot shows a positive attractivity effect of quality museums over longer distances, which is greatly reduced for shorter trips. This result would imply that such museums have a greater attraction on far-away tourists. Consistently with this finding, the spatial competition effect measured by L.CultDem dest appears to vary over distance as well, as it becomes virtually null for the shortest distance class.

Our general result, then, can be summarized in the emergence of inferential evidence on the role of distance in determining the attractiveness of cultural offer. This role appears to be particularly true when evaluating destination regions and the related spatial competition for tourists, as distance strengthens such direct and indirect pull effects. 

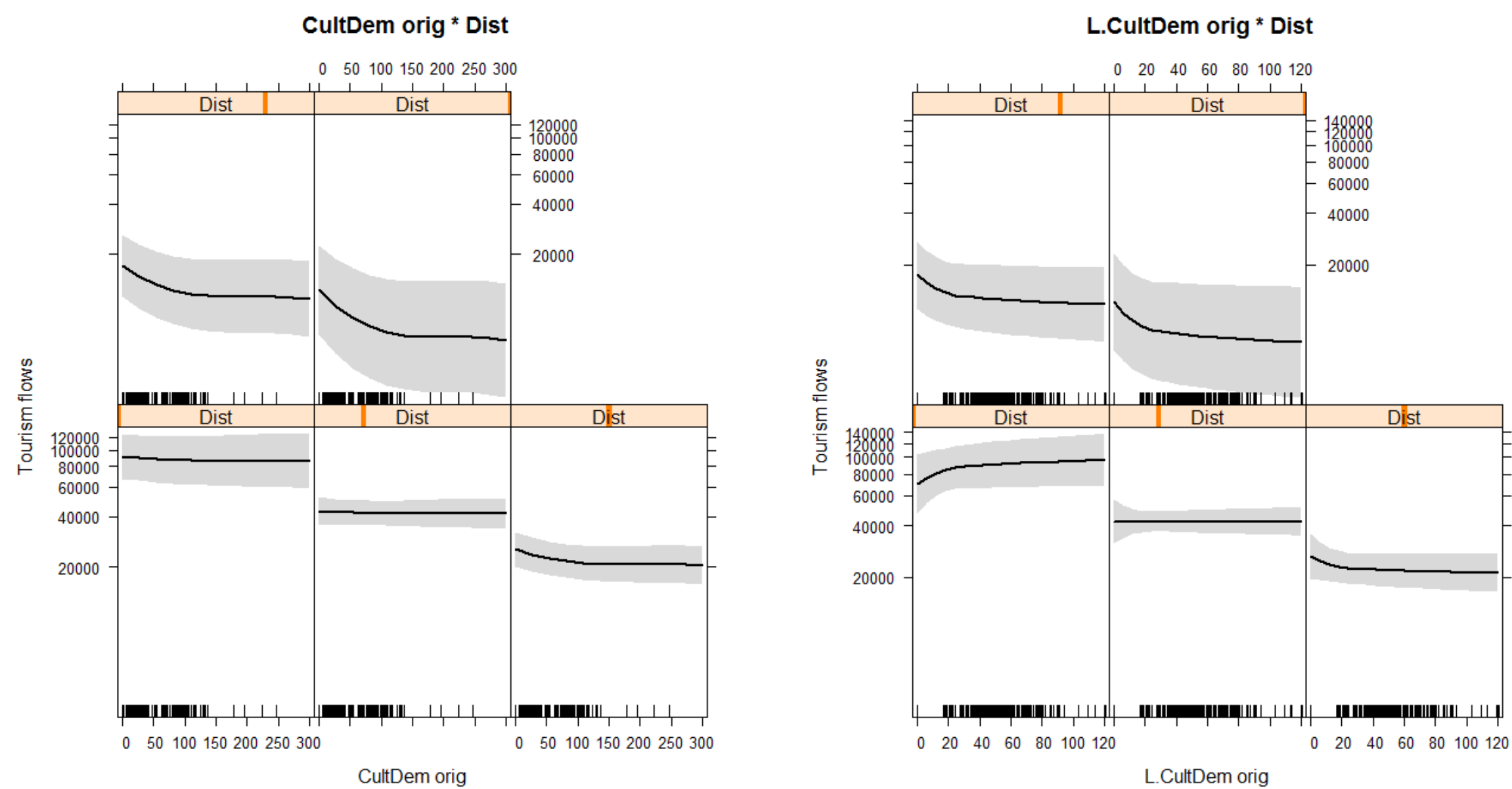

Figure 1. Marginal effects for the interaction of Dist with CultDem orig and L.CultDem orig 

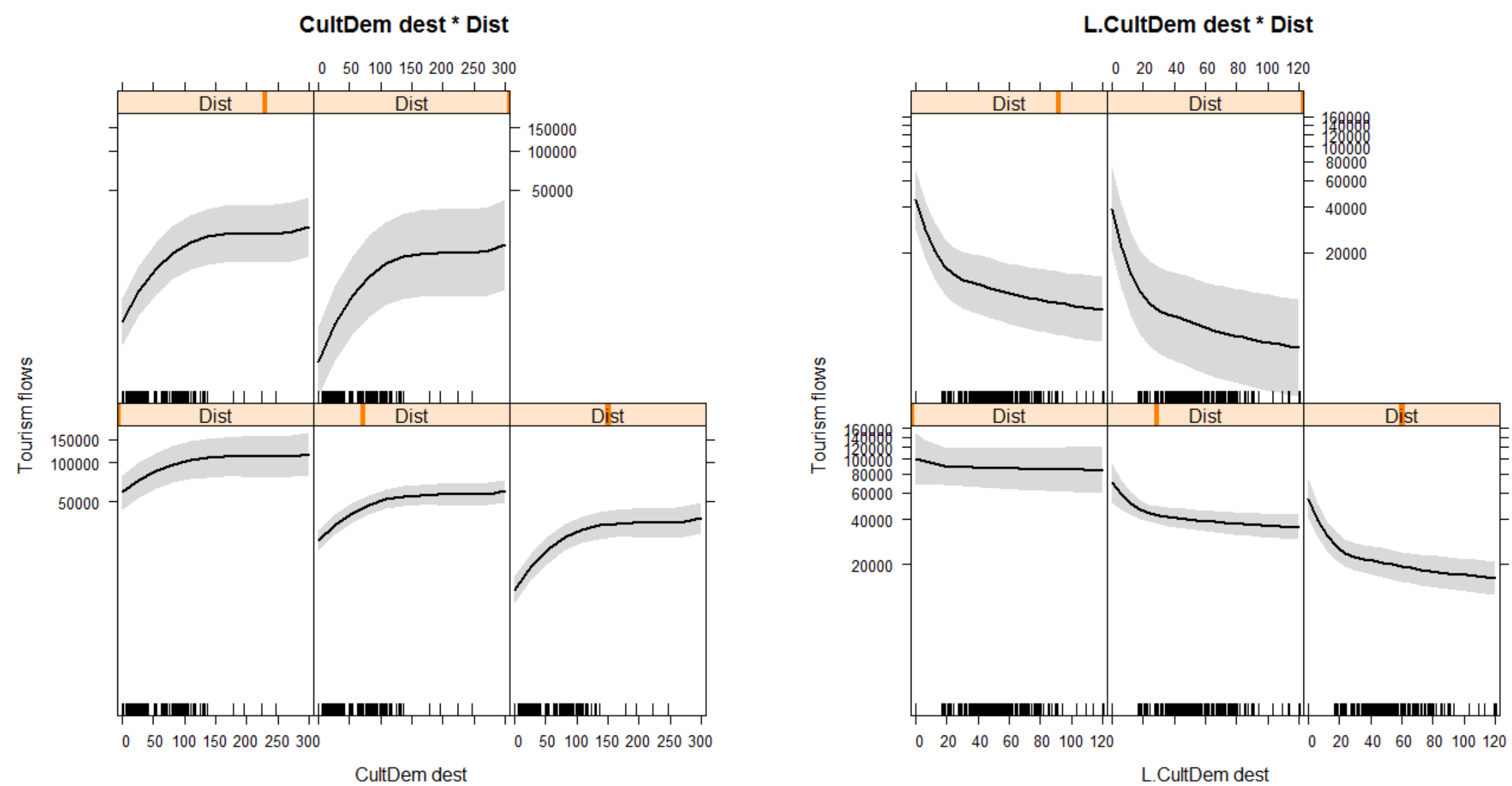

Figure 2. Marginal effects for the interaction of Dist with CultDem dest and L.CultDem dest 


\section{Concluding Remarks}

In this paper, we have analysed the relationship between domestic tourism, cultural offer, and the distance covered by tourists. Our empirical application, based on a spatial interaction model for the 20 Italian regions over the years 1998-2009, has shown that geographical distance between origin and destination regions plays a non-trivial role in determining the relevance of cultural offer.

In particular, the following results emerge from our analysis:

- for the origin regions, the (negative) effect of cultural offer on tourism outflows (i.e., on emissivity) increases over distance, so that more cultural offer 'near home' (either in your residence region or in nearby ones) disincentivates travel less to distant destinations;

- for the destination regions, the (positive) effect of cultural offer on tourism inflows (i.e., on attractivity) increases over distance, meaning that the direct benefits obtainable from providing a cultural offer are greater in attracting more distant tourists. On the flipside, spatial competition (substitution effects) intensifies over distance as well, rendering the overall pull effect of cultural offer ambiguous.

In summary, we show that cultural offer does influence the tourist's willingness to travel, and that this effect is mediated by geographical distance: (i) when available 'near home', cultural offer inhibits greater geographical mobility, most likely due to substitution with excursionism or other non-recordable forms of tourism; (ii) when available 'on site', cultural offer incentivates greater geographical mobility. Such findings, which could be found to be apparently contradicting, may be interpreted by reflecting on the different role of distance in tourism in comparison, for example, to industrial or trade economics. In the latter, distance is only seen as a cost. In tourism, instead, the travelling distance is at the same time a cost and a utility (as suggested by the cubic polynomial found for distance in Patuelli et al., 2013). This interpretation is consistent with the concept of a circular (rather than linear) tourism space, in which increasing distance has an incentive or deterrence effect depending on individual preferences (e.g., slow and fast tourists, which can be imagined to travel clockwise and anticlockwise along the circular tourism space). Further research is of course needed in order to verify more in depth this interpretation and its implications, in particular from a theoretical perspective.

\section{References}

Candela, G., Mussoni, M., and Patuelli, R. (2014) Centralized vs Decentralized Tourism Policies: a Spatial Interaction Model Framework. Paper presented at the 10th World Congress of the RSAI, Ayutthaya.

Cuccia, T., and Rizzo, I. (2011) Tourism Seasonality in Cultural Destinations: Empirical Evidence from Sicily. Tourism Management, 32(3), 589-595.

Cuccia, T., and Cellini, R. (2007) Is Cultural Heritage Really Important for Tourists? A Contingent Rating Study. Applied Economics, 39(2), 261-271.

Dritsakis, N. (2004) Cointegration Analysis of German and British Tourism Demand for Greece. Tourism Management, 25(1), 111-119.

Fotheringham, A.S. (1983) A New Set of Spatial-Interaction Models: The Theory of Competing Destinations. Environment and Planning A, 15, 15-36.

Haynes, K.E., and Fotheringham, A.S. (1984) Gravity and Spatial Interaction Models, Sage Publications: Beverly Hills. 
Herbert, D. (2001) Literary Places, Tourism and the Heritage Experience. Annals of Tourism Research, 28(2), 312-333.

Khadaroo, J., and Seetanah, B. (2008) The Role of Transport Infrastructure in International Tourism Development: A Gravity Model Approach. Tourism Management, 29(5), 831840.

Lim, C. (1997) Review of International Tourism Demand Models. Annals of Tourism Research, 24(4), 835-849.

Massidda, C., and Etzo, I. (2011) The Determinants of Italian Domestic Tourism: A Panel Data Analysis. Tourism Management, (forthcoming).

Patuelli, R., Mussoni, M., and Candela, G. (2013) The Effects of World Heritage Sites on Domestic Tourism: A Spatial Interaction Model for Italy. Journal of Geographical Systems, 15(3), 369-402.

Santos Silva, J.M.C., and Tenreyro, S. (2006) The Log of Gravity. Review of Economics and Statistics, 88(4), 641-658.

Sen, A., and Smith, T.E. (1995) Gravity Models of Spatial Interaction Behavior, Springer: Heidelberg and New York.

Sheldon, P.J., and Var, T. (1985) Tourism Forecasting: A Review of Empirical Research. Journal of Forecasting, 4(2), 183-195.

Uysal, M., and Crompton, J.L. (1985) An Overview of Approaches Used to Forecast Tourism Demand. Journal of Travel Research, 23(4), 7-15.

Vietze, C. (2008) Cultural Effects on Inbound Tourism into the USA: A Gravity Approach, Jena Economic Research Papers No. 2008 - 037). Jena: Friedrich Schiller University and Max Planck Institute of Economics. Document Number)

Witt, S.F., and Witt, C.A. (1995) Forecasting Tourism Demand: A Review of Empirical Research. International Journal of Forecasting, 11(3), 447-475. 


\section{Appendix}

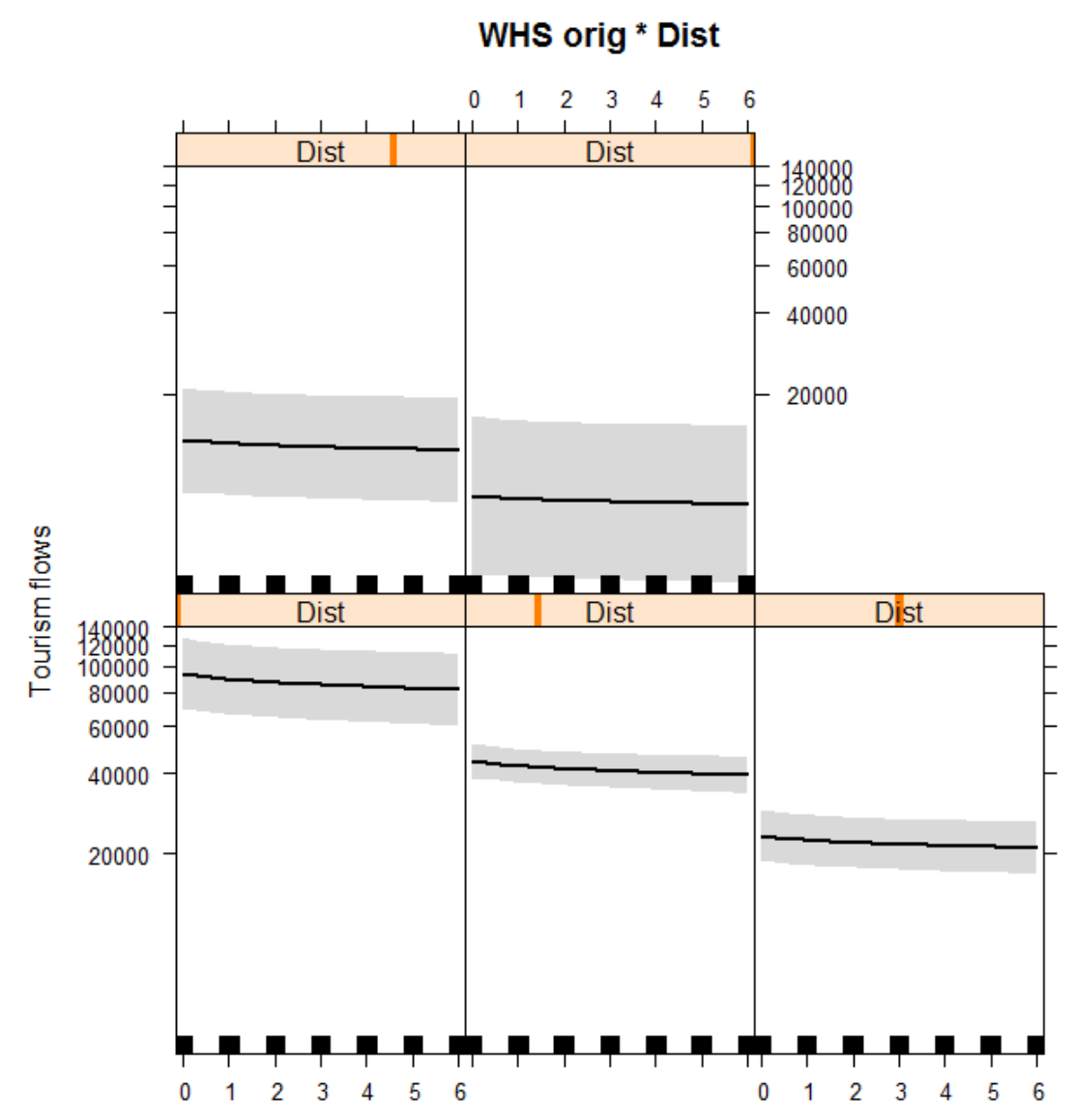

WHS orig

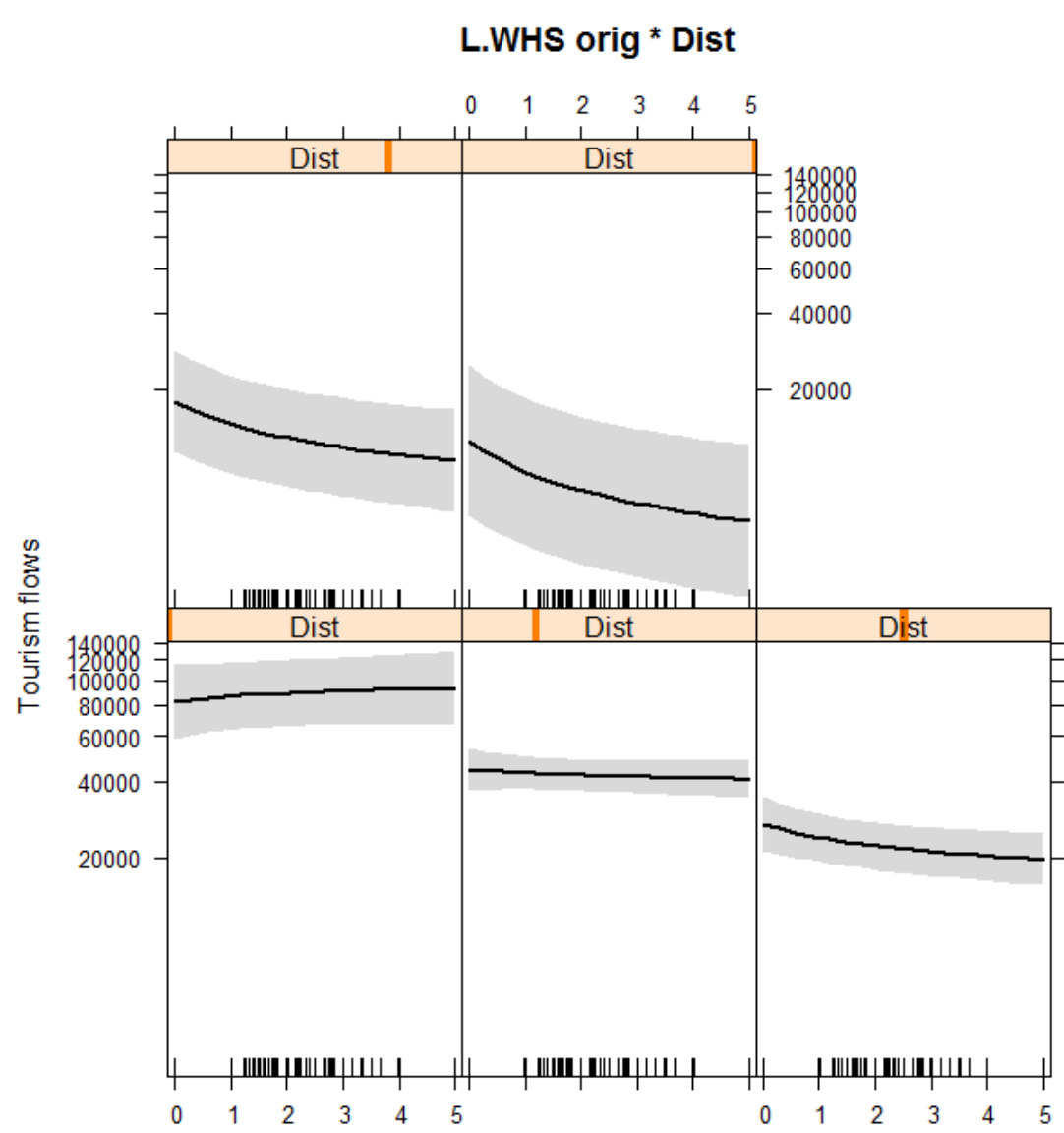

L.WHS orig

Figure A.1. Marginal effects for the interaction of Dist with WHS orig and L.WHS orig 

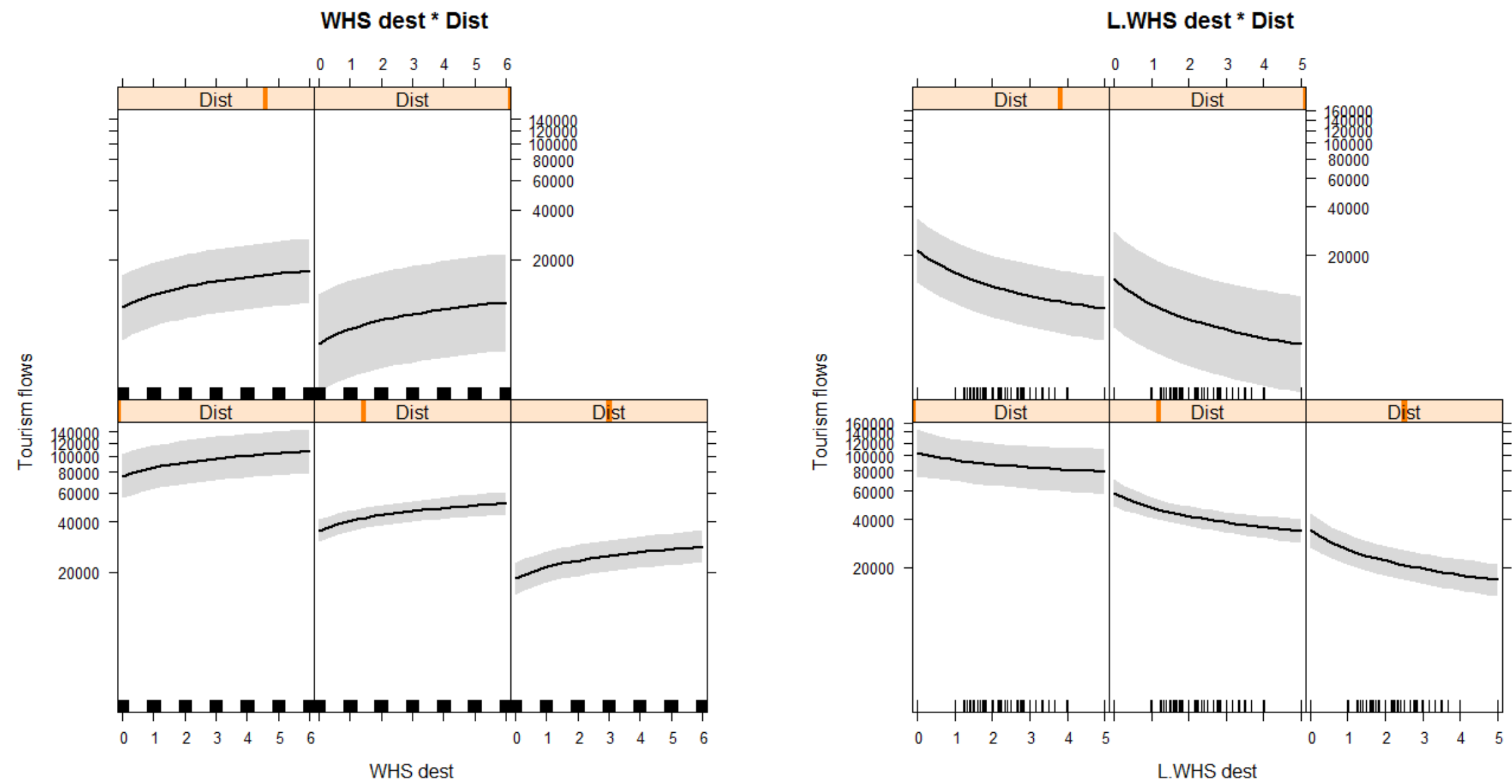

Figure A.2. Marginal effects for the interaction of Dist with WHS dest and L.WHS dest 


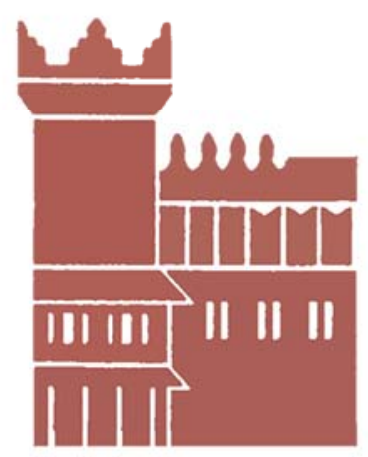

Alma Mater Studiorum - Università di Bologna DEPARTMENT OF ECONOMICS

Strada Maggiore 45

40125 Bologna - Italy

Tel. +39051 2092604

Fax +390512092664

http://www.dse.unibo.it 